\title{
Простейшие комбинаторные типы выпуклых полиэдров с кристаллографическими группами симметрии
}

\author{
Войтеховский Ю.Л. ${ }^{1}$, Степенщиков Д.Г. ${ }^{2}$ \\ ${ }^{1}$ Санкт-Петербургский горный университет,Voytekhovskiy_Yul@pers.spmi.ru \\ ${ }^{2}$ Геологический институт КНЦ РАH, stepen@geoksc.apatity.ru
}

Аннотация. Для каждой из 32 кристаллографических групп симметрии найден комбинаторный тип выпуклого полиэдра с минимальным числом граней.

Ключевые слова: Комбинаторный тип, кристаллографическая группа симметрии, полиэдр.

\section{The simplest combinatorial types of the convex polyhedra with crystallographic symmetry groups}

\author{
Voytekhovsky Yu.L. ${ }^{1}$, Stepenshchikov D.G. ${ }^{2}$ \\ ${ }^{1}$ Saint Petersburg Mining University,Voytekhovskiy_Yul@pers.spmi.ru \\ ${ }^{2}$ Geological Institute Kola SC RAS, stepen@geoksc.apatity.ru
}

\begin{abstract}
For each of the 32 crystallographic symmetry groups the combinatorial type of the convex polyhedron with a minimum number of faces was found.

Key words: Combinatorial type, crystallographic symmetry group, polyhedron.
\end{abstract}

Грани, вершины и ребра любого выпуклого полиэдра образуют вершинно-реберный каркас, который также называется его комбинаторным типом (далее - комбинаторный тип). Формально он представляет собой трехсвязный планарный граф, характеризуемый некоторой группой автоморфизмов. По теореме Мани (Mani, 1971; Klaus, 2010, p. 28-2), для такого графа существует выпуклый полиэдр, группа симметрии которого изоморфна группе автоморфизмов графа. Эта группа симметрии максимальна для всех полиэдров данного комбинаторного типа. Так, среди полиэдров с комбинаторным типом куба (6 4-угольных граней, сходящихся по 3 в каждой из 8 вершин) - ромбоэдров, параллелепипедов и т.д., самый симметричный именно куб $(m-3 m)$. Назовем такую максимальную группу симметрии комбинаторной. Ранее авторами получены все комбинаторные типы выпуклых 4- ... 12-эдров и простых (в каждой вершине сходятся ровно 3 грани) 13- ... 16-эдров (Войтеховский, Степенщиков, $2008 \mathrm{a}, \mathrm{b}$ ), охарактеризованные комбинаторными группами симметрии.

Одна и та же комбинаторная симметрия может быть у различных комбинаторных типов, имеющих, в общем случае, разное число граней (вершин, ребер). Отсюда возникает задача поиска первого появления данной группы симметрии на множестве комбинаторных типов, иначе, задача поиска комбинаторного типа с заданной группой симметрии и минимальным числом граней. Назовем такой комбинаторный тип и соответствующий ему максимально симметричный полиэдр простейшими.

С точки зрения минералогии особый интерес представляют 32 кристаллографические группы симметрии. Анализ известного многообразия комбинаторных типов выявил простейшие комбинаторные типы для 24 кристаллографических групп симметрии: $1,-1,2, m, 3,222, m m 2,4,-4,2 / m$, 32,-6, 3m, 4mm, mmm, -42m, -6m2, -3m, 6mm, 23, 4/mmm, 6/mmm, -43m, m-3m. Оставшиеся 8 групп симметрии $(m-3,432,422,4 / m,-3,6,622,6 / m)$, очевидно, реализуются среди комбинаторных типов с бо́льшим числом граней, но генерирование их огромного $\left(\sim 10^{8}\right)$ многообразия для отыскания всего 8 штук трудоемко и неэффективно.

В статье (Войтеховский, Степенщиков, 2019) для оставшихся групп симметрии указаны найденные конструктивным путем простейшие комбинаторные типы. Чтобы подтвердить результат, разработан специальный метод доказательства. Он заключается в следующем: предположим, что существует комбинаторный тип с меньшим числом граней и данной комбинаторной группой симметрии. Тогда, по теореме Мани, существует выпуклый полиэдр с данной группой симметрии. Его грани принадлежат простым формам, выводимым с помощью элементов симметрии группы. 

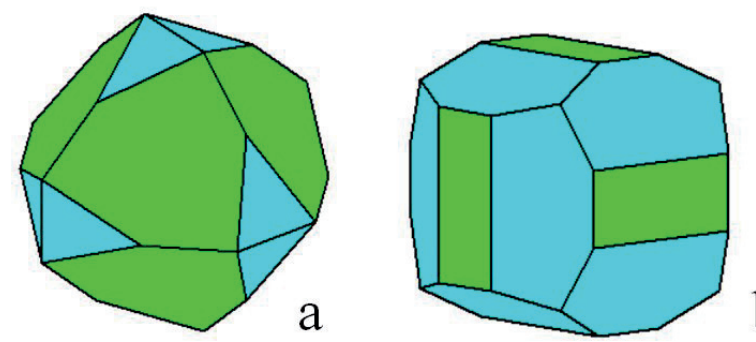

Рис. 1. $\mathrm{a}$ - простейший комбинаторный тип с комбинаторной симметрией $m-3, \mathrm{~b}$ - комбинация куба и пентагондодекаэдра с комбинаторной симметрией $m-3 m$.

Fig. 1. a - the simplest combinatorial type with combinatorial symmetry $m-3, \mathrm{~b}-$ the combination of cube and penb tagonal dodecahedron with combinatorial symmetry $m-3 m$.

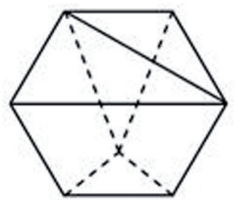

$1, \mathrm{~F}=7, \mathrm{~V}=7$

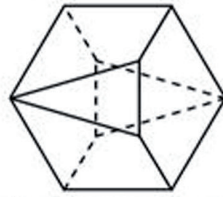

$2 / m, \mathrm{~F}=8, \mathrm{~V}=10$

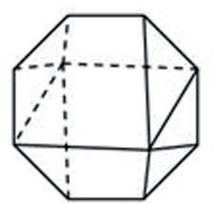

$-1, \mathrm{~F}=10, \mathrm{~V}=10$

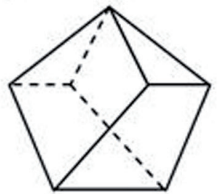

$m m 2, \mathrm{~F}=6, \mathrm{~V}=7$

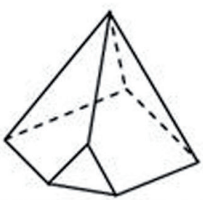

$m, \mathrm{~F}=6, \mathrm{~V}=7$

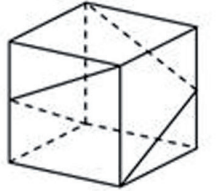

$222, \mathrm{~F}=10, \mathrm{~V}=10$

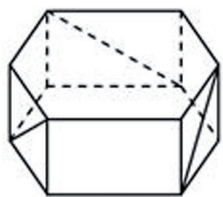

$32, \mathrm{~F}=11, \mathrm{~V}=12$

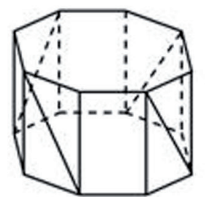

422, $\mathrm{F}=14, \mathrm{~V}=16$

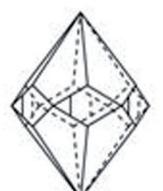

$6 / \mathrm{m}, \mathrm{F}=24, \mathrm{~V}=32$

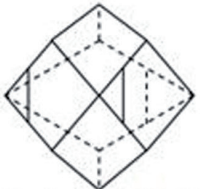

$-6, F=12, V=17$

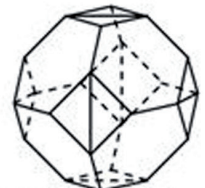

$m-3, \mathrm{~F}=20, \mathrm{~V}=24$

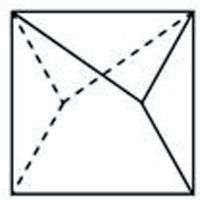

$2, \mathrm{~F}=6, \mathrm{~V}=6$

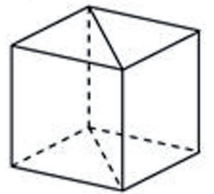

$m m m, \mathrm{~F}=8, \mathrm{~V}=8$

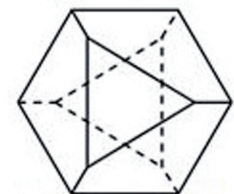

$-3 m, \mathrm{~F}=8, \mathrm{~V}=12$

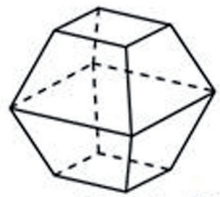

$4 / \mathrm{mmm}, \mathrm{F}=10, \mathrm{~V}=12$

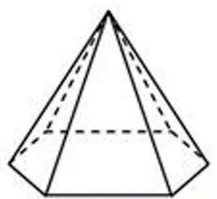

$6 \mathrm{~mm}, \mathrm{~F}=7, \mathrm{~V}=7$

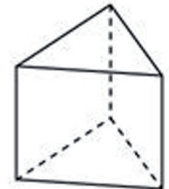

$-6 m 2, \mathrm{~F}=5, \mathrm{~V}=6$

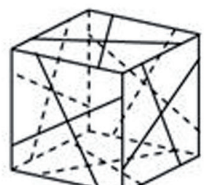

$432, \mathrm{~F}=24, \mathrm{~V}=38$

Рис. 2. Простейшие комбинаторные типы выпуклых полиэдров для 32 кристаллографических групп симметрии.

Fig. 2. The simplest combinatorial types of convex polyhedra for the 32 crystallographic symmetry groups. 
Рассмотрим наборы простых форм, образующие все возможные комбинаторно различные полиэдры с числом граней, меньшим, чем у исходного. Если комбинаторный тип каждого из них имеет комбинаторную симметрию, отличную от данной, то он не может быть для последней простейшим, и первоначальное предположение неверно. Метод хорошо работает для групп симметрии высокого порядка, когда положение и количество граней сокращает число возможных комбинаций. Далее мы ограничимся доказательством для комбинаторных типов кубической сингонии. Покажем, что для группы $m-3$ простейший комбинаторный тип имеет 20 граней и реализуется комбинацией октаэдра и пентагондодекаэдра (рис. 1 a).

Для данной группы симметрии простыми формами являются куб, ромбододекаэдр, пентагондодекаэдры, октаэдр, тригон- и тетрагонтриоктаэдры, дидодекаэдры. Рассмотрим их возможные комбинации, число граней которых больше 12 (известное многообразие) и меньше 20. Их три: куб с октаэдром (14 граней), куб с ромбододекаэдром (18 граней) и куб с пентагондодекаэдром (18 граней). Первые две порождают полиэдры с недопустимыми в группе $m-3$ осями 4 порядка, т.е. их комбинаторные типы имеют заведомо более высокую симметрию. Последняя порождает комбинаторно идентичные полиэдры с комбинаторной группой симметрии $m-3 m$ (рис. 1 b). Следовательно, комбинаторный тип на рис. 1 а простейший для группы симметрии $m-3$.

Докажем, что для группы симметрии 432 простейший комбинаторный тип имеет 24 грани и реализуется пентагонтриоктаэдром. Здесь простыми формами являются куб, ромбододекаэдр, тетрагексаэдры, октаэдр, тригон-, тетрагон- и пентагонтриоктаэдры. Рассмотрим их возможные комбинации, число граней которых больше 12 и меньше 24. Их три: куб с октаэдром (14 граней), куб с ромбододекаэдром (18 граней) и октаэдр с ромбододекаэдром (20 граней). Все они порождают полиэдры с недопустимыми в группе 432 плоскостями симметрии. Таким образом, полиэдра с комбинаторной симметрией 432 и числом граней менее 24 нет. Следовательно, комбинаторный тип пентагонтриоктаэдра действительно является простейшим для симметрии 432. Отметим, что тетрагексаэдры, тригон- и тетрагонтриоктаэдры имеют плоскости симметрии и поэтому их комбинаторные типы не могут быть простейшими для группы симметрии 432, хотя они тоже 24-гранники.

Аналогично доказываются и случаи оставшихся 6 кристаллографических групп симметрии. Они рассмотрены нами в общем виде для бесконечного множества групп симметрии средней категории в статье (Степенщиков, Войтеховский, 2020). Там же с целью исчерпания многообразия точечных групп симметрии рассмотрены икосаэдрические группы -3-5m и 235. В результате составлен полный список простейших комбинаторных типов для всех 32 кристаллографических групп симметрии (рис. 2). Группа симметрии 23 представлена комбинаторным типом непростого полиэдра - он получен «подгонкой» известного простого 16-гранника с целью уменьшения количества вершин.

На рисунке 1. простейший комбинаторный тип для группы симметрии 1 - один из двух возможных, а простейший комбинаторный тип для группы симметрии $3 m$ имеет дуальный тип, также простейший для данной группы. Отметим, что лишь минимальные полиэдры трёх групп симметрии высшей категории $(-43 m, m-3 m, 432)$ представлены одной простой формой; все остальные - комбинации нескольких форм. Их максимальное число - 7 моноэдров - наблюдается у минимального полиэдра примитивной группы симметрии.

\section{Литература}

1. Войтеховский Ю.Л., Степенщиков Д.Г. Комбинаторная кристалломорфология. Кн. IV. Выпуклые полиэдры. Т. І. 4- ... 12-эдры. Апатиты: КНЦ РАН. 2008 а. 833 с.

2. Войтеховский Ю.Л., Степенщиков Д.Г. Комбинаторная кристалломорфология. Кн. IV. Выпуклые полиэдры. Т. ІІ. Простые 13- ... 16-эдры. Апатиты: КНЦ РАН. 2008 b. 828 с.

3. Войтеховский Ю.Л., Степенщиков Д.Г. О простейших выпуклых полиэдрах точечных групп комбинаторной симметрии 422, 4/m, $-3,6,622,6 / m, m-3,432 / /$ Математические исследования в естественных науках. Tp. XVI Всерос. научн. школы. Апатиты, Геол. ин-т КНЦ РАН, 22 окт. 2019 г. Апатиты: Изд-во K \& M. 2019. С. 29-33.

4. Степенщиков Д.Г., Войтеховский Ю.Л. Простейшие комбинаторные типы выпуклых полиэдров с группами симметрии средней сингонии и икосаэдрическими группами симметрии $-3-5 m$ и $235 / / \mathrm{Tp}$. XVI Bсерос. Ферсмановской научн. сессии. Апатиты, Геол. ин-т КНЦ РАН, 5-8 апр. 2020 г. Апатиты: Изд-во КНЦ РАН, 2020. (Наст. сб.)

5. Klaus D. Sattler Handbook of Nanophysics: Clusters and Fullerenes. New York: CRC Press, 2010. 909 p.

6. Mani P. Automorphismen von polyedrischen Graphen // Math. Ann. 1971. N 192. S. 279-303. 